Pacific

Journal of

Mathematics

\title{
UNFAITHFUL COMPLEX HYPERBOLIC TRIANGLE GROUPS, I: INVOLUTIONS
}

JOHN R. PARKER 


\title{
UNFAITHFUL COMPLEX HYPERBOLIC TRIANGLE GROUPS, I: INVOLUTIONS
}

\author{
JOHN R. PARKER
}

\begin{abstract}
A complex hyperbolic triangle group is the group of complex hyperbolic isometries generated by complex involutions fixing three complex lines in complex hyperbolic space. Such a group is called equilateral if there is an isometry of order three that cyclically permutes the three complex lines. We consider equilateral triangle groups for which the product of each pair of involutions and the product of all three involutions are all nonloxodromic. We classify all such groups that are discrete.
\end{abstract}

\section{Introduction}

A complex hyperbolic triangle group is a group generated by three complex reflections that fix complex lines in complex hyperbolic space. Unlike real reflections, complex reflections can be of arbitrary order. Much of the literature is confined to the case where the reflections have order two. In this paper we consider that case and in the subsequent paper [Parker and Paupert 2007] we consider the case where the generators have higher order.

The study of complex hyperbolic triangle groups was begun in [Goldman and Parker 1992] where ideal triangle groups were considered. Since then there have been many developments. There have been two strands to this work. First, following [Goldman and Parker 1992], discrete and faithful representations of triangle groups have been investigated; see [Schwartz 2001] for example. On the other hand, there has been the study of discrete representations where certain group elements are required to be elliptic of finite order; see [Deraux 2006; Falbel and Parker 2006; Parker 2006; Schwartz 2003] for example. These representations are necessarily unfaithful. Schwartz [2002] has given an excellent survey that outlines progress and gives a conjectural overview of what we might expect. A more recent survey is also contained in [Pratoussevitch 2005]. Pratoussevitch also considers the case where the generators are complex reflections of higher order, which is related

MSC2000: 20H10, 22E40, 51M10.

Keywords: complex hyperbolic geometry, triangle group.

This work was carried out during a visit to Université Pierre et Marie Curie (Paris VI) supported by CNRS. 
to earlier work of Mostow; see [Mostow 1980] for example. We will treat groups with generators of higher order in the sequel to this paper [Parker and Paupert 2007].

Three complex lines $L_{1}, L_{2}$ and $L_{3}$ in complex hyperbolic space form an equilateral triangle if each pair intersects and if there is a symmetry map $J$ of order 3 in $\mathrm{SU}(2,1)$ so that $J\left(L_{j}\right)=L_{j+1}$ (with indices taken cyclically). For $j=1,2,3$ let $I_{j}$ be the complex reflection of order 2 fixing $L_{j}$. Then

$$
I_{2}=J I_{1} J^{-1} \quad \text { and } \quad I_{3}=J I_{2} J^{-1}=J^{-1} I_{1} J .
$$

We call the group $\Delta=\left\langle I_{1}, I_{2}, I_{3}\right\rangle$ an equilateral triangle group. In this case $\Delta$ is a normal subgroup of $\Gamma=\left\langle I_{1}, J\right\rangle$. At first sight, it might appear that there is a full $S_{3}$ symmetry group inside $\mathrm{SU}(2,1)$ operating here, but this is only the case when $\Delta$ preserves a Lagrangian plane. The symmetry preserving $L_{1}$ but interchanging $L_{2}$ and $L_{3}$ is antiholomorphic. We shall have more to say about this in Section 2.2.

Our starting point is the following theorem, proved in [Schwartz 2001, Section 3.3]:

Theorem 1.1 [Schwartz 2001]. Let $\Delta=\left\langle I_{1}, I_{2}, I_{3}\right\rangle$ be the group of complex hyperbolic isometries generated by complex involutions $I_{j}$ each fixing a complex line. Suppose that there is a symmetry map $J$ of order 3 so that

$$
I_{2}=J I_{1} J^{-1}, \quad \text { and } I_{3}=J^{-1} I_{1} J .
$$

If $I_{1} I_{2}$ is parabolic and $I_{1} I_{2} I_{3}$ is elliptic then $\Delta$ is not discrete.

The main theorem of this paper is to consider equilateral triangle groups $\Delta=$ $\left\langle I_{1}, I_{2}, I_{3}\right\rangle$ where $I_{1} I_{2}$ and $I_{1} I_{2} I_{3}$ are both elliptic. Clearly $I_{2} I_{3} I_{1}$ and $I_{3} I_{1} I_{2}$ are conjugate to $I_{1} I_{2} I_{3}$ and so are elliptic; the fact that $\Delta$ is equilateral means that both $I_{2} I_{3}$ and $I_{3} I_{1}$ are conjugate to $I_{1} I_{2}$ and hence are elliptic; the fact that each $I_{j}$ is an involution means that $I_{2} I_{1}=\left(I_{1} I_{2}\right)^{-1}$ and $I_{3} I_{2} I_{1}=\left(I_{1} I_{2} I_{3}\right)^{-1}$, and so both of these maps are elliptic as well. We classify all such $\Delta$ that are discrete and we find that there are remarkably few of them. The point is that $I_{1} I_{2}$ and $I_{1} I_{2} I_{3}$ should simultaneously have finite order. We now give a rough statement of our main theorem. For a more precise statement see Theorem 3.7 and Proposition 4.5.

Theorem 1.2. Let $\Delta=\left\langle I_{1}, I_{2}, I_{3}\right\rangle$ be the group of complex hyperbolic isometries generated by complex involutions $I_{j}$ each fixing a complex line. Suppose that there is a symmetry map $J$ of order 3 so that $I_{2}=J I_{1} J^{-1}$ and $I_{3}=J^{-1} I_{1} J$. Suppose that $I_{1} I_{2}$ and $I_{1} I_{2} I_{3}$ are both elliptic. Then $\Delta$ is discrete if and only if one of the following is true:

(i) $\Delta$ is finite;

(ii) $\Delta$ is a normal subgroup of one of Livné's lattices; 
(iii) $\Delta$ is Deraux's lattice, with $I_{1} I_{2}$ of order 4 and $I_{1} I_{2} I_{3}$ of order 10 ;

(iv) $\Delta$ is the group described in Section 4.2, with $I_{1} I_{2}$ of order 14 and $I_{1} I_{2} I_{3}$ of order 14.

There are other possible theorems along these lines. According to Schwartz's conjectural picture [2002] discreteness of $\Delta=\left\langle I_{1}, I_{2}, I_{3}\right\rangle$ is controlled by whether $I_{1} I_{2} I_{3}$ and $I_{1} I_{2} I_{1} I_{3}$ are nonelliptic (for equilateral triangle groups, by symmetry $I_{1} I_{2} I_{1} I_{3}$ is elliptic if and only if each of $I_{2} I_{3} I_{2} I_{1}$ and $I_{3} I_{1} I_{3} I_{2}$ are elliptic). We could have considered the case of equilateral triangle groups where $I_{1} I_{2}$ and $I_{1} I_{2} I_{1} I_{3}$ are elliptic of finite order. Either using the formulae of Pratoussevitch [2005] or using the formulae of Section 2.3 we find that $\operatorname{tr}\left(I_{1} I_{2}\right)=|\tau|^{2}-1$ and $\operatorname{tr}\left(I_{1} I_{2} I_{1} I_{3}\right)=\left|\tau^{2}-\bar{\tau}\right|^{2}-1$. Choosing $\tau$ so that $|\tau|=2 \cos \frac{\pi}{n}$ and $\left|\tau^{2}-\bar{\tau}\right|=2 \cos \frac{\pi}{m}$ yields groups for which $I_{1} I_{2}$ has order $n$ and $I_{1} I_{2} I_{1} I_{3}$ has order $m$. From this it is easy to see that if $I_{1} I_{2} I_{3}$ is loxodromic and $I_{1} I_{2} I_{1} I_{3}$ is elliptic then $1<|\tau|^{2}<\frac{11}{3}$, which is equivalent to $4 \leq n \leq 10$. Conjecturally, for a given $n$ in this range these groups are discrete for all sufficiently large values of $m$; compare the remark just after Theorem 4.7 of [Schwartz 2002]. (See also the second remark on [Schwartz 2007, p. 8].) Schwartz [2003] proves the discreteness of the group of this type with $n=4$ and $m=7$. A similar proof should work for $n=4$ and $m \geq 8$. Note that when $n=4$ and $m=5$ we obtain Deraux's lattice, in which case $I_{1} I_{2} I_{3}$ is elliptic and has order 10 .

After finishing this paper, Julien Paupert and I were discussing complex hyperbolic equilateral triangle groups where the generators have higher order. It turns out that we can use the same equations to discuss discreteness of these groups. Certain values of $\tau$ yield some of Mostow's groups [1980] and other values of $\tau$ give normal subgroups of Mostow's groups. This simultaneously generalizes Theorem 1.2 (ii), since Livné's lattices are examples of Mostow's groups, and work of Sauter [1990] on commensurability between Mostow's groups. There are also sporadic groups when the generators have higher order. The details may be found in [Parker and Paupert 2007].

\section{Parameters and traces}

In this section we show how to parametrise equilateral complex hyperbolic triangle groups with a single complex parameter $\tau$ and we find the values of $\tau$ that correspond to such a group. We then describe how the properties of the group (for example the type of $I_{1} I_{2}$ and $I_{1} I_{2} I_{3}$ ) vary with $\tau$. We will try to keep this account as self-contained as possible. However, we shall assume a certain amount of background knowledge of complex hyperbolic geometry. For such background material on complex hyperbolic space see [Goldman 1999] and for material on complex hyperbolic triangle groups see [Schwartz 2002] or [Pratoussevitch 2005]. 
2.1. Complex reflections. Let $L_{1}$ be a complex line in complex hyperbolic 2-space $\mathbf{H}_{\mathbb{C}}^{2}$ and write $I_{1}$ for the complex reflection of order 2 fixing $L_{1}$. We may lift $I_{1}$ to a matrix in $\mathrm{SU}(2,1)$. If the polar vector of $L_{1}$ is $\mathbf{n}_{1}$ then $I_{1}$ is given by

$$
I_{1}(\mathbf{z})=-\mathbf{z}+2 \frac{\left\langle\mathbf{z}, \mathbf{n}_{1}\right\rangle}{\left\langle\mathbf{n}_{1}, \mathbf{n}_{1}\right\rangle} \mathbf{n}_{1} .
$$

In [Goldman 1999, Section 3.3.2], the polar vectors of two complex lines are used to determine the geometry of their relative position.

Proposition 2.1 [Goldman 1999]. Suppose that $L_{1}$ and $L_{2}$ are complex lines in $\mathbf{H}_{\mathbb{C}}^{2}$ with polar vectors $\mathbf{n}_{1}$ and $\mathbf{n}_{2}$. Let

$$
\mathcal{N}\left(L_{1}, L_{2}\right)=\frac{\left\langle\mathbf{n}_{1}, \mathbf{n}_{2}\right\rangle\left\langle\mathbf{n}_{2}, \mathbf{n}_{1}\right\rangle}{\left\langle\mathbf{n}_{1}, \mathbf{n}_{1}\right\rangle\left\langle\mathbf{n}_{2}, \mathbf{n}_{2}\right\rangle} .
$$

(i) If $\mathcal{N}\left(L_{1}, L_{2}\right)>1$ then $L_{1}$ and $L_{2}$ are ultraparallel;

(ii) if $\mathcal{N}\left(L_{1}, L_{2}\right)=1$ then either $L_{1}$ and $L_{2}$ are asymptotic or $L_{1}=L_{2}$;

(iii) if $\mathcal{N}\left(L_{1}, L_{2}\right)<1$ then $L_{1}$ and $L_{2}$ intersect with angle $\theta$ where $\mathcal{N}\left(L_{1}, L_{2}\right)=$ $\cos ^{2} \theta$.

We shall be interested in equilateral triangle groups $\Delta=\left\langle I_{1}, I_{2}, I_{3}\right\rangle$ generated by complex involutions fixing complex lines $L_{1}, L_{2}$ and $L_{3}$. The hypothesis that the triangle is equilateral means there is a symmetry map $J$ of order 3 in $\mathrm{SU}(2,1)$ so that $J\left(L_{j}\right)=L_{j+1}$, where the indices are taken modulo 3 . This implies that $J\left(\mathbf{n}_{j}\right)=\mathbf{n}_{j+1}$ and so

$$
\mathcal{N}\left(L_{j}, L_{j+1}\right)=\frac{\left\langle\mathbf{n}_{j}, \mathbf{n}_{j+1}\right\rangle\left\langle\mathbf{n}_{j+1}, \mathbf{n}_{j}\right\rangle}{\left\langle\mathbf{n}_{j}, \mathbf{n}_{j}\right\rangle\left\langle\mathbf{n}_{j+1}, \mathbf{n}_{j+1}\right\rangle}=\frac{\left|\left\langle J\left(\mathbf{n}_{j}\right), \mathbf{n}_{j}\right\rangle\right|^{2}}{\left\langle\mathbf{n}_{j}, \mathbf{n}_{j}\right\rangle^{2}} .
$$

2.2. The parameter space. Suppose we are given an equilateral triangle of complex lines $L_{1}, L_{2}$ and $L_{3}$ with polar vectors $\mathbf{n}_{1}, \mathbf{n}_{2}$ and $\mathbf{n}_{3}$ satisfying $J\left(\mathbf{n}_{j}\right)=\mathbf{n}_{j+1}$ where $j=1,2,3$ taken modulo 3 . Because $J$ preserves the Hermitian form, $\left\langle\mathbf{n}_{j}, \mathbf{n}_{j}\right\rangle$ is the same positive real number for each $j$. We normalize $\mathbf{n}_{j}$ so that this number is 2. Likewise $\left\langle\mathbf{n}_{j+1}, \mathbf{n}_{j}\right\rangle=\left\langle J\left(\mathbf{n}_{j}\right), \mathbf{n}_{j}\right\rangle$ is the same complex number for each $j$ which we define to be $\tau$. That is,

$$
\left\langle\mathbf{n}_{1}, \mathbf{n}_{1}\right\rangle=\left\langle\mathbf{n}_{2}, \mathbf{n}_{2}\right\rangle=\left\langle\mathbf{n}_{3}, \mathbf{n}_{3}\right\rangle=2, \quad\left\langle\mathbf{n}_{2}, \mathbf{n}_{1}\right\rangle=\left\langle\mathbf{n}_{3}, \mathbf{n}_{2}\right\rangle=\left\langle\mathbf{n}_{1}, \mathbf{n}_{3}\right\rangle=\tau .
$$

Let $H$ be the matrix of the Hermitian form, that is, $\langle\mathbf{z}, \mathbf{w}\rangle=\mathbf{w}^{*} H \mathbf{z}$. We define $N$ to be the matrix whose columns are $\mathbf{n}_{1}, \mathbf{n}_{2}, \mathbf{n}_{3}$. Then the $i j$-th entry of $N^{*} H N$ is $\left\langle\mathbf{n}_{j}, \mathbf{n}_{i}\right\rangle$ and so

$$
N^{*} H N=\left(\begin{array}{ccc}
2 & \tau & \bar{\tau} \\
\bar{\tau} & 2 & \tau \\
\tau & \bar{\tau} & 2
\end{array}\right)
$$


Lemma 2.2. Let $L_{1}, L_{2}$ and $L_{3}$ be complex lines in $\mathbf{H}_{\mathbb{C}}^{2}$ with polar vectors $\mathbf{n}_{1}, \mathbf{n}_{2}$ and $\mathbf{n}_{3}$. Suppose that the Hermitian products of these vectors satisfy (2-2). Then the vectors $\mathbf{n}_{1}, \mathbf{n}_{2}$ and $\mathbf{n}_{3}$ are linearly independent if and only if

$$
8+2 \operatorname{Re}\left(\tau^{3}\right)-6|\tau|^{2} \neq 0
$$

Proof. We have

$$
8+2 \operatorname{Re}\left(\tau^{3}\right)-6|\tau|^{2}=\operatorname{det}\left(\begin{array}{lll}
2 & \tau & \bar{\tau} \\
\bar{\tau} & 2 & \tau \\
\tau & \bar{\tau} & 2
\end{array}\right)=\operatorname{det}\left(N^{*} H N\right)=\operatorname{det}(H)|\operatorname{det}(N)|^{2} .
$$

Since $\operatorname{det}(H) \neq 0$ we see that $8+2 \operatorname{Re}\left(\tau^{3}\right)-6|\tau|^{2} \neq 0$ if and only if $N$ is nonsingular.

Let us now consider the case where the vectors $\mathbf{n}_{1}, \mathbf{n}_{2}$ and $\mathbf{n}_{3}$ are linearly independent. We consider the other case at the end of the section. Following [Mostow 1980, p. 214], we choose coordinates so that

$$
\mathbf{n}_{1}=\left(\begin{array}{l}
1 \\
0 \\
0
\end{array}\right), \quad \mathbf{n}_{2}=\left(\begin{array}{l}
0 \\
1 \\
0
\end{array}\right), \quad \mathbf{n}_{3}=\left(\begin{array}{l}
0 \\
0 \\
1
\end{array}\right) .
$$

In this case, the matrix $N$ (whose columns are the vectors $\mathbf{n}_{j}$ ) is the identity. Hence, using (2-3), we see that the Hermitian form must be $\langle\mathbf{z}, \mathbf{w}\rangle=\mathbf{w}^{*} H_{\tau} \mathbf{z}$ where

$$
H_{\tau}=\left(\begin{array}{ccc}
2 & \tau & \bar{\tau} \\
\bar{\tau} & 2 & \tau \\
\tau & \bar{\tau} & 2
\end{array}\right)
$$

We can immediately write down $J$ and, using (2-1), the involutions $I_{j}$. They are

$$
J=\left(\begin{array}{lll}
0 & 0 & 1 \\
1 & 0 & 0 \\
0 & 1 & 0
\end{array}\right), I_{1}=\left(\begin{array}{ccc}
1 & \tau & \bar{\tau} \\
0 & -1 & 0 \\
0 & 0 & -1
\end{array}\right), I_{2}=\left(\begin{array}{ccc}
-1 & 0 & 0 \\
\bar{\tau} & 1 & \tau \\
0 & 0 & -1
\end{array}\right), I_{3}=\left(\begin{array}{ccc}
-1 & 0 & 0 \\
0 & -1 & 0 \\
\tau & \bar{\tau} & 1
\end{array}\right) .
$$

From this it is clear that the groups $\Gamma=\left\langle I_{1}, J\right\rangle$ and $\Delta=\left\langle I_{1}, I_{2}, I_{3}\right\rangle$ are completely determined, up to conjugation, by the parameter $\tau$. However, not all values of $\tau$ correspond to complex hyperbolic triangle groups: it may be that the Hermitian matrix $H_{\tau}$ does not have signature $(2,1)$. We now determine this by finding the eigenvalues of $H_{\tau}$. In this lemma and throughout the paper we write $\omega=e^{2 \pi i / 3}=$ $\frac{1}{2}(-1+i \sqrt{3})$.

Lemma 2.3. Let $H_{\tau}$ be given by (2-4) and write $\tau=t+i$ s. The eigenvalues of $H_{\tau}$ are

$2+\tau+\bar{\tau}=2+2 t, \quad 2+\tau \bar{\omega}+\bar{\tau} \omega=2-t+\sqrt{3} s, \quad 2+\tau \omega+\bar{\tau} \bar{\omega}=2-t-\sqrt{3} s$. 
Proof. We observe that eigenvectors for $H_{\tau}$ are

$$
\left(\begin{array}{l}
1 \\
1 \\
1
\end{array}\right), \quad\left(\begin{array}{l}
1 \\
\bar{\omega} \\
\omega
\end{array}\right), \quad\left(\begin{array}{l}
1 \\
\omega \\
\bar{\omega}
\end{array}\right) .
$$

Their eigenvalues are

$$
2+\tau+\bar{\tau}, \quad 2+\tau \bar{\omega}+\bar{\tau} \omega \text { and } 2+\tau \omega+\bar{\tau} \bar{\omega},
$$

respectively.

Corollary 2.4. The matrix $H_{\tau}$ has signature $(2,1)$ if and only if

$$
6|\tau|^{2}-\tau^{3}-\bar{\tau}^{3}-8>0
$$

Proof. It is easy to check (for example by adding them) that all three eigenvalues cannot be negative. Thus $H_{\tau}$ has signature $(2,1)$ if and only if its determinant is negative. That is

$$
0>(2+\tau+\bar{\tau})(2+\tau \bar{\omega}+\bar{\tau} \omega)(2+\tau \omega+\bar{\tau} \bar{\omega})=8+\tau^{3}+\bar{\tau}^{3}-6|\tau|^{2} .
$$

We now describe how the signature of $H_{\tau}$ varies as $\tau$ varies in $\mathbb{C}$. There are three lines each of which is the locus where one of the eigenvalues vanishes. In Figure 1 we have drawn these three lines. These lines have seven complementary regions in $\mathbb{C}$, which fall into three types:

- The central triangle where all three eigenvalues are positive and so $H_{\tau}$ has signature $(3,0)$, that is it is positive definite.

- Three infinite components each sharing a common edge with the central triangle. In these regions two eigenvalues are positive and one negative and so $H_{\tau}$ has signature $(2,1)$. This is our parameter space.

- Three infinite components that each only abuts the central triangle in a point. Here one eigenvalue is positive and two are negative and so $H_{\tau}$ has signature $(1,2)$. These correspond to groups of complex hyperbolic isometries generated by three complex involutions that each fix a point.

The values of $\tau$ satisfying (2-5) make up our parameter space. This space has three components related by multiplication by powers of $\omega=e^{2 \pi i / 3}$. This ambiguity corresponds to the choice we have made when lifting symmetry in $\mathrm{PU}(2,1)$ to the corresponding matrix $J$ in $\mathrm{SU}(2,1)$, the triple cover of $\mathrm{PU}(2,1)$. In other words, the ordered triples $\left\{\mathbf{n}_{1}, \omega \mathbf{n}_{2}, \bar{\omega} \mathbf{n}_{3}\right\}$ and $\left\{\mathbf{n}_{1}, \bar{\omega} \mathbf{n}_{2}, \omega \mathbf{n}_{3}\right\}$ correspond to the same group as $\left\{\mathbf{n}_{1}, \mathbf{n}_{2}, \mathbf{n}_{3}\right\}$. Hence $\tau$ is only defined up to a cube root of unity. Factoring out by this equivalence, our parameter space is in bijection with one of the three components where $H_{\tau}$ has signature $(2,1)$, which we described above. There is a 


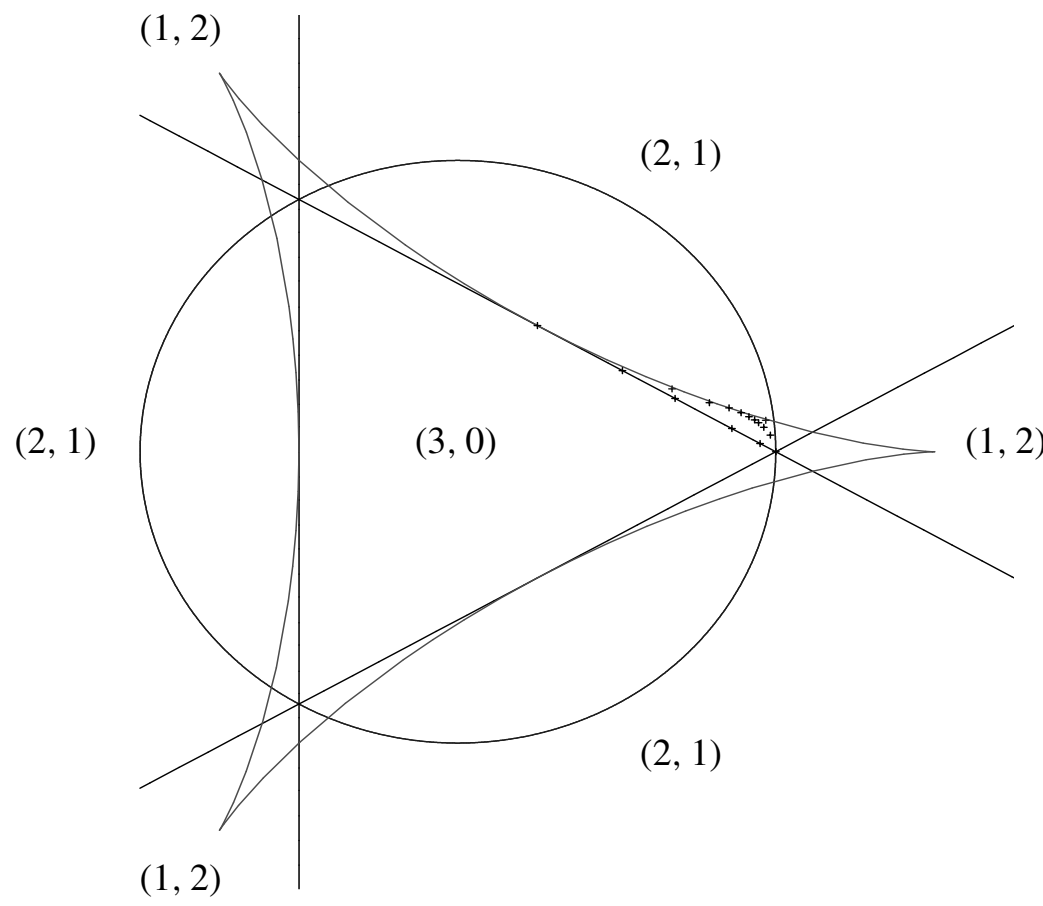

Figure 1. The parameter space. We have drawn the points corresponding to the groups listed in Theorem 1.2. For more details of this part of the picture, see Figure 2.

further symmetry of our setup, namely complex conjugating $\tau$. This corresponds to sending $\tau=\left\langle\mathbf{n}_{j+1}, \mathbf{n}_{j}\right\rangle$ to $\bar{\tau}=\left\langle\mathbf{n}_{j}, \mathbf{n}_{j+1}\right\rangle$. Up to conjugation, this preserves $I_{1}$ and sends $J$ to $J^{-1}$. In particular, it swaps the roles of $I_{2}$ and $I_{3}$. Thus all the symmetries in $S_{3}$ (acting on $L_{1}, L_{2}$ and $L_{3}$ ) either preserve $\tau$ or send it to $\bar{\tau}$. Our parameter space also respects this symmetry. Hence we may restrict our attention to those $\tau$ whose argument lies in $\left[0, \frac{\pi}{3}\right]$. In fact, this description of the parameter space can be shown to be a reformulation in terms of $\tau$ of [Pratoussevitch 2005, Proposition 1], for our special case. We will give details of how to pass from $\tau$ to Pratoussevitch's parameters in the next section.

We conclude this section by considering the case where the vectors $\mathbf{n}_{j}$ are linearly dependent, that is, when $N$ is singular. Using Lemma 2.2, this implies that

$$
0=8+\tau^{3}+\bar{\tau}^{3}-6|\tau|^{2}=(2+\tau+\bar{\tau})(2+\tau \bar{\omega}+\bar{\tau} \omega)(2+\tau \omega+\bar{\tau} \bar{\omega}) .
$$

It will be convenient to make a choice of which one of these linear factors is zero. In Section 4.1 below it will be useful to suppose that $\tau \omega+\bar{\tau} \bar{\omega}=-2$ and so we focus on that case here. We shall also explain how to obtain the formulae in the other two cases. We begin with a geometrical description of the complex lines $L_{1}$, $L_{2}$ and $L_{3}$. 
Proposition 2.5. Let $L_{1}, L_{2}$ and $L_{3}$ be complex lines in $\mathbf{H}_{\mathbb{C}}^{2}$ with polar vectors $\mathbf{n}_{1}$, $\mathbf{n}_{2}$ and $\mathbf{n}_{3}$. Suppose that the Hermitian products of these vectors satisfy (2-2). Then

(i) $8+2 \operatorname{Re}\left(\tau^{3}\right)-6|\tau|^{2}=0$ and $|\tau|<2$ if and only if $L_{1}, L_{2}$ and $L_{3}$ have a unique point of intersection in $\mathbf{H}_{\mathbb{C}}^{2}$;

(ii) $8+2 \operatorname{Re}\left(\tau^{3}\right)-6|\tau|^{2}=0$ and $|\tau|=2$ if and only if $L_{1}, L_{2}$ and $L_{3}$ coincide;

(iii) $8+2 \operatorname{Re}\left(\tau^{3}\right)-6|\tau|^{2}=0$ and $|\tau|>2$ if and only if there is a complex line $L^{\perp}$ in $\mathbf{H}_{\mathbb{C}}^{2}$ that is orthogonal to each of $L_{1}, L_{2}$ and $L_{3}$.

Proof. From Lemma 2.2 we see that $8+2 \operatorname{Re}\left(\tau^{3}\right)-6|\tau|^{2}=0$ if and only the matrix $N$ defined above is singular. This is true if and only if there exists a nonzero vector $\mathbf{z}_{0}$ so that

$$
\mathbf{0}=N^{*} H \mathbf{z}_{0}=\left(\begin{array}{c}
\left\langle\mathbf{z}_{0}, \mathbf{n}_{1}\right\rangle \\
\left\langle\mathbf{z}_{0}, \mathbf{n}_{2}\right\rangle \\
\left\langle\mathbf{z}_{0}, \mathbf{n}_{3}\right\rangle
\end{array}\right)
$$

Therefore $\mathbf{z}_{0}$ satisfies

$$
\left\langle\mathbf{z}_{0}, \mathbf{n}_{1}\right\rangle=\left\langle\mathbf{z}_{0}, \mathbf{n}_{2}\right\rangle=\left\langle\mathbf{z}_{0}, \mathbf{n}_{3}\right\rangle=0 .
$$

Hence $\mathbf{z}_{0}$ either corresponds to a common intersection point of $L_{1}, L_{2}$ and $L_{3}$ or to the polar vector of a common orthogonal complex line. Which of these possibilities occurs depends on whether $\mathcal{N}\left(L_{1}, L_{2}\right)=\frac{|\tau|^{2}}{4}$ is greater than, equal to or less than 1, using Proposition 2.1. This proves (i) and (iii).

In order to prove the result, all that remains is to consider the case when $|\tau|=2$ and to decide whether the complex lines are asymptotic or coincide. In this case

$$
0=8+2 \operatorname{Re}\left(\tau^{3}\right)-6|\tau|^{2}=2 \operatorname{Re}\left(\tau^{3}\right)-16
$$

and so $\tau^{3}=8$ and so $\frac{\tau}{2}$ is a cube root of unity. In this case the matrix $N$ has rank 1 and so $N^{*} H$ has a two-dimensional kernel. The projection of this kernel is $L_{1}=L_{2}=L_{3}$, proving (ii).

We can reinterpret Proposition 2.5 in terms of the group $\Delta=\left\langle I_{1}, I_{2}, I_{3}\right\rangle$. This group fixes a point of $\mathbf{H}_{\mathbb{C}}^{2}$, that is it is elementary, if and only if $8+2 \operatorname{Re}\left(\tau^{3}\right)-6|\tau|^{2}$ vanishes and $|\tau| \leq 2$. If such a group is discrete then it must be finite. In particular, the case $|\tau|=2$ corresponds to the order 2 group where $I_{1}=I_{2}=I_{3}$. On the other hand, $\Delta$ preserves a complex line if and only if $8+2 \operatorname{Re}\left(\tau^{3}\right)-6|\tau|^{2}$ vanishes and $|\tau|>2$. If such a group is discrete then it is Fuchsian.

When $|\tau| \neq 2$, that is in the cases given by Proposition 2.5 (i) and (iii), we can once again choose a basis and use this to write down the Hermitian form and matrix representatives for $I_{1}, I_{2}, I_{3}$ and $J$. Suppose that $\mathbf{n}_{1}, \mathbf{n}_{2}, \mathbf{n}_{3}$ satisfy (2-2). As in the proof of Proposition 2.5, there exists $\mathbf{z}_{0}$ satisfying (2-6). As indicated in that proof, this vector is negative when $|\tau|<2$ and positive when $|\tau|>2$ (recall we 
have excluded the case of $|\tau|=2$ ). Thus, without loss of generality, we suppose that

$$
\left\langle\mathbf{z}_{0}, \mathbf{z}_{0}\right\rangle=|\tau|-2
$$

Let $N^{\prime}$ be the matrix whose columns are $\mathbf{n}_{1}, \mathbf{n}_{2}, \mathbf{z}_{0}$. As our Hermitian form is nondegenerate, it is clear that $\mathbf{z}_{0}$ is not in the span of $\mathbf{n}_{1}$ and $\mathbf{n}_{2}$. Therefore $\left\{\mathbf{n}_{1}, \mathbf{n}_{2}, \mathbf{z}_{0}\right\}$ is a basis of $\mathbb{C}^{3}$ and so $N^{\prime}$ is nondegenerate. Moreover, if $H$ is the matrix of the Hermitian form then

$$
N^{\prime *} H N^{\prime}=\left(\begin{array}{ccc}
2 & \tau & 0 \\
\bar{\tau} & 2 & 0 \\
0 & 0 & |\tau|-2
\end{array}\right)
$$

We choose coordinates so that

$$
\mathbf{n}_{1}=\left(\begin{array}{l}
1 \\
0 \\
0
\end{array}\right), \quad \mathbf{n}_{2}=\left(\begin{array}{l}
0 \\
1 \\
0
\end{array}\right), \quad \mathbf{z}_{0}=\left(\begin{array}{l}
0 \\
0 \\
1
\end{array}\right)
$$

Hence the matrix $N^{\prime}$ is the identity, and our Hermitian form is given by the following matrix $H_{\tau}^{\prime}$, which only depends on $\tau$ :

$$
H_{\tau}^{\prime}=\left(\begin{array}{ccc}
2 & \tau & 0 \\
\bar{\tau} & 2 & 0 \\
0 & 0 & |\tau|-2
\end{array}\right)
$$

Notice that $H_{\tau}^{\prime}$ has eigenvalues $2+|\tau|, 2-|\tau|$ and $|\tau|-2$ and so it has signature $(2,1)$ whenever $|\tau| \neq 2$. Once more, we can use (2-1) to find $I_{1}$ and $I_{2}$. They are

$$
I_{1}=\left(\begin{array}{ccc}
1 & \tau & 0 \\
0 & -1 & 0 \\
0 & 0 & -1
\end{array}\right), \quad I_{2}=\left(\begin{array}{ccc}
-1 & 0 & 0 \\
\bar{\tau} & 1 & 0 \\
0 & 0 & -1
\end{array}\right)
$$

Using $\tau \omega+\bar{\tau} \bar{\omega}=-2$, one may easily find $\mathbf{n}_{3}$ and this allows us to write down $I_{3}$ and $J$ :

$$
\mathbf{n}_{3}=\left(\begin{array}{c}
-\omega \\
-\bar{\omega} \\
0
\end{array}\right), \quad I_{3}=\left(\begin{array}{ccc}
1+\bar{\tau} \bar{\omega} & -\bar{\tau} \omega & 0 \\
-\tau \bar{\omega} & 1+\tau \omega & 0 \\
0 & 0 & -1
\end{array}\right), \quad J=\left(\begin{array}{ccc}
0 & -\omega & 0 \\
1 & -\bar{\omega} & 0 \\
0 & 0 & \bar{\omega}
\end{array}\right)
$$

Once again, it is clear that the groups $\Gamma=\left\langle I_{1}, J\right\rangle$ and $\Delta=\left\langle I_{1}, I_{2}, I_{3}\right\rangle$ are completely determined up to conjugation by the parameter $\tau$. In the remaining two cases, namely $\tau \bar{\omega}+\bar{\tau} \omega=-2$ and $\tau+\bar{\tau}=-2$, the formulae for $\mathbf{n}_{3}, I_{3}$ and $J$ are obtained from (2-7) by swapping $\omega$ and $\bar{\omega}$ or by replacing both $\omega$ and $\bar{\omega}$ by 1 , respectively. 
2.3. Traces. We have seen that if $\tau$ satisfies (2-5) then $\tau$ corresponds to a group $\Gamma=\left\langle I_{1}, J\right\rangle$, in $\operatorname{SU}(2,1)$, with an equilateral triangle group $\Delta=\left\langle I_{1}, I_{2}, I_{3}\right\rangle$ as an index three normal subgroup. In this section we write down the traces of certain elements of $\Gamma$ in terms of $\tau$. This should be compared to [Pratoussevitch 2005, Theorem 9] where formulae for the traces of elements in $\Delta$ that are integer polynomials in $|\tau|^{2}, \tau^{3}$ and $\bar{\tau}^{3}$ are given (in our language). We could write down the traces directly from our expressions for $J, I_{1}, I_{2}$ and $I_{3}$. We choose to give a more general argument as this is more illuminating and is independent of our choice of Hermitian form.

Lemma 2.6. Let $A$ be any element of $\mathrm{SU}(2,1)$. Then

$$
\operatorname{tr}\left(I_{1} A\right)=-\operatorname{tr}(A)+2 \frac{\left\langle A\left(\mathbf{n}_{1}\right), \mathbf{n}_{1}\right\rangle}{\left\langle\mathbf{n}_{1}, \mathbf{n}_{1}\right\rangle} .
$$

Proof. Using (2-1) we see that

$$
I_{1} A(\mathbf{z})=-A(\mathbf{z})+2 \frac{\left\langle A(\mathbf{z}), \mathbf{n}_{1}\right\rangle}{\left\langle\mathbf{n}_{1}, \mathbf{n}_{1}\right\rangle} \mathbf{n}_{1} .
$$

We must find the trace of the matrix corresponding to the linear map

$$
T: \mathbf{z} \mapsto\left\langle A(\mathbf{z}), \mathbf{n}_{1}\right\rangle \mathbf{n}_{1} .
$$

Writing $\langle\mathbf{z}, \mathbf{w}\rangle=\mathbf{w}^{*} H \mathbf{z}$ and using the fact that this is a complex scalar, we have

$$
\left\langle A(\mathbf{z}), \mathbf{n}_{1}\right\rangle \mathbf{n}_{1}=\mathbf{n}_{1} \mathbf{n}_{1}^{*} H A \mathbf{z}=\left(\mathbf{n}_{1}\left(A^{*} H \mathbf{n}_{1}\right)^{*}\right) \mathbf{z} .
$$

Hence the matrix of $T$ is $\mathbf{n}_{1}\left(A^{*} H \mathbf{n}_{1}\right)^{*}$. Now if a matrix can be written in the form $\mathbf{u v}^{*}$ for column vectors $\mathbf{u}$ and $\mathbf{v}$, then its trace is just $\mathbf{v}^{*} \mathbf{u}$. Thus

$$
\operatorname{tr}\left(\mathbf{n}_{1}\left(A^{*} H \mathbf{n}_{1}\right)^{*}\right)=\left(A^{*} H \mathbf{n}_{1}\right)^{*} \mathbf{n}_{1}=\mathbf{n}_{1}^{*} H A \mathbf{n}_{1}=\left\langle A\left(\mathbf{n}_{1}\right), \mathbf{n}_{1}\right\rangle .
$$

Hence

$$
\operatorname{tr}\left(I_{1} A\right)=-\operatorname{tr} A+2 \frac{\operatorname{tr}\left(\mathbf{n}_{1}\left(A^{*} H \mathbf{n}_{1}\right)^{*}\right)}{\left\langle\mathbf{n}_{1}, \mathbf{n}_{1}\right\rangle}=-\operatorname{tr} A+2 \frac{\left\langle A\left(\mathbf{n}_{1}\right), \mathbf{n}_{1}\right\rangle}{\left\langle\mathbf{n}_{1}, \mathbf{n}_{1}\right\rangle} .
$$

Corollary 2.7. Let $I_{1}$ be a complex involution fixing a complex line $L_{1}$ with polar vector $\mathbf{n}_{1}$. Let $J \in \mathrm{SU}(2,1)$ be a regular elliptic map of order 3 . Then

$$
\operatorname{tr}\left(I_{1} J\right)=2 \frac{\left\langle J\left(\mathbf{n}_{1}\right), \mathbf{n}_{1}\right\rangle}{\left\langle\mathbf{n}_{1}, \mathbf{n}_{1}\right\rangle}
$$

Proof. This follows directly from the previous lemmas using the fact that, since $J$ is regular elliptic of order three, its trace is zero.

Using (2-2) and the fact that $\mathbf{n}_{j+1}=J\left(\mathbf{n}_{j}\right)$, an immediate consequence of Corollary 2.7 is that $\operatorname{tr}\left(I_{j} J\right)=\tau$. This fact is our justification for naming our parameter $\tau$. The following theorem is just a restatement of [Goldman 1999, Theorem 6.2.4]. 
Proposition 2.8. Let $\tau$ be given by (2-2). Then $I_{1} J$ is regular elliptic if and only if

$$
|\tau|^{4}-4 \tau^{3}-4 \bar{\tau}^{3}+18|\tau|^{2}-27<0 .
$$

The curve given by the equality in Proposition 2.8 is a deltoid; see Figure 1. Groups for which $I_{1} J$ is regular elliptic correspond to points in the interior of this deltoid. Points on the deltoid correspond to points where $I_{1} J$ is either a complex reflection or is parabolic. Since $I_{1} I_{2} I_{3}=\left(I_{1} J\right)^{3}$ we can determine the type of $I_{1} I_{2} I_{3}$ from $I_{1} J$. (Note that it may be that $I_{1} J$ is regular elliptic and that $I_{1} I_{2} I_{3}$ is a complex reflection.)

We now consider $I_{1} I_{2}$, and hence by symmetry $I_{2} I_{3}$ and $I_{3} I_{1}$ as well.

Proposition 2.9. Let $L_{1}$ and $L_{2}$ be complex lines in $\mathbf{H}_{\mathbb{C}}^{2}$ with polar vectors $\mathbf{n}_{1}$ and $\mathbf{n}_{2}$ respectively. Suppose that $\left\langle\mathbf{n}_{1}, \mathbf{n}_{1}\right\rangle=\left\langle\mathbf{n}_{2}, \mathbf{n}_{2}\right\rangle=2$ and $\left\langle\mathbf{n}_{2}, \mathbf{n}_{1}\right\rangle=\tau$. Let $I_{1}$ and $I_{2}$ denote the complex involutions fixing $L_{1}$ and $L_{2}$. Then

$$
\operatorname{tr}\left(I_{1} I_{2}\right)=|\tau|^{2}-1 .
$$

Proof. Let $\mathbf{z}_{2}$ and $\mathbf{z}_{3}$ be any distinct vectors on $L_{1}$ (for example, if the fixed point of $J$ is not on $L_{1}$, we could choose $\mathbf{z}_{2}=L_{1} \cap L_{3}$ and $\left.\mathbf{z}_{3}=L_{1} \cap L_{2}\right)$. Then $\left\{\mathbf{n}_{1}, \mathbf{z}_{2}, \mathbf{z}_{3}\right\}$ is a basis for $\mathbb{C}^{2,1}$. We write $\mathbf{n}_{2}$ in terms of this basis as

$$
\mathbf{n}_{2}=\alpha \mathbf{n}_{1}+\beta \mathbf{z}_{2}+\gamma \mathbf{z}_{3} .
$$

Since $\mathbf{n}_{1}$ is orthogonal to $\mathbf{z}_{2}$ and $\mathbf{z}_{3}$ then $\left\langle\mathbf{n}_{2}, \mathbf{n}_{1}\right\rangle=\alpha\left\langle\mathbf{n}_{1}, \mathbf{n}_{1}\right\rangle$ and so $\alpha=\frac{\tau}{2}$. Since $\mathbf{n}_{1}$ is a 1-eigenvector for $I_{1}$ and $\mathbf{z}_{2}, \mathbf{z}_{3}$ are both (-1)-eigenvectors we have

$$
\mathbf{n}_{2}=\frac{\tau}{2} \mathbf{n}_{1}+\beta \mathbf{z}_{2}+\gamma \mathbf{z}_{3}, \quad I_{1}\left(\mathbf{n}_{2}\right)=\frac{\tau}{2} \mathbf{n}_{1}-\beta \mathbf{z}_{2}-\gamma \mathbf{z}_{3} .
$$

Adding these two expressions we see that $I_{1}\left(\mathbf{n}_{2}\right)=\tau \mathbf{n}_{1}-\mathbf{n}_{2}$. Therefore

$$
\left\langle I_{1}\left(\mathbf{n}_{2}\right), \mathbf{n}_{2}\right\rangle=\tau\left\langle\mathbf{n}_{1}, \mathbf{n}_{2}\right\rangle-\left\langle\mathbf{n}_{2}, \mathbf{n}_{2}\right\rangle=\frac{|\tau|^{2}}{2}\left\langle\mathbf{n}_{2}, \mathbf{n}_{2}\right\rangle-\left\langle\mathbf{n}_{2}, \mathbf{n}_{2}\right\rangle .
$$

Hence using Lemma 2.6 we have

$$
\operatorname{tr}\left(I_{2} I_{1}\right)=-\operatorname{tr}\left(I_{1}\right)+2 \frac{\left\langle I_{1}\left(\mathbf{n}_{2}\right), \mathbf{n}_{2}\right\rangle}{\left\langle\mathbf{n}_{2}, \mathbf{n}_{2}\right\rangle}=1+|\tau|^{2}-2=|\tau|^{2}-1 .
$$

If an element of $\mathrm{SU}(2,1)$ with real trace is elliptic then its trace lies in $[-1,3)$ and conversely any element of $\mathrm{SU}(2,1)$ whose trace lies in the real interval $(-1,3)$ is elliptic. If the trace is -1 it is either elliptic or parabolic. Hence we see that $I_{1} I_{2}$ is elliptic if and only if $|\tau|<2$. In Figure 1 we show how the circle $|\tau|=2$ compares to the deltoid of Proposition 2.8. Furthermore, we can now see that Pratoussevitch's parameters [2005] may be written in terms of $\tau$ as

$$
r_{1}=r_{2}=r_{3}=\frac{|\tau|}{2}, \quad \alpha=\arg \left(\tau^{3}\right) .
$$


Using Proposition 2.1 we can, in fact, relate the trace of $I_{1} I_{2}$ to the relative position of $I_{1}$ and $I_{2}$.

Corollary 2.10. Let $L_{1}$ be a complex line in $\mathbf{H}_{\mathbb{C}}^{2}$ with polar vector $\mathbf{n}_{1}$. Let $J \in$ $\mathrm{SU}(2,1)$ and write $L_{2}=J\left(L_{1}\right)$. Let $I_{1}$ and $I_{2}$ denote the complex involutions fixing $L_{1}$ and $L_{2}$. Then

(i) if $L_{1}$ and $L_{2}$ are ultraparallel then $\operatorname{tr}\left(I_{1} I_{2}\right)>3$;

(ii) if $L_{1}$ and $L_{2}$ are asymptotic then $\operatorname{tr}\left(I_{1} I_{2}\right)=3$;

(iii) if $L_{1}$ and $L_{2}$ intersect with angle $\theta$ then $\operatorname{tr}\left(I_{1} I_{2}\right)=2 \cos (2 \theta)+1$.

\section{When $I_{1} I_{2} I_{3}$ is elliptic and $I_{1} I_{2}$ is nonloxodromic}

This section is the heart of the paper. We restrict our attention to those groups for which $I_{1} I_{2} I_{3}$ is elliptic of finite order and $I_{1} I_{2}$ is either elliptic of finite order or else parabolic. These are groups for which $\tau$ lies inside or on the deltoid and inside or on the circle in Figure 1. Since they have finite order, the eigenvalues of $I_{1} I_{2} I_{3}$ and $I_{1} I_{2}$ are all roots of unity (in the case where $I_{1} I_{2}$ is parabolic then its eigenvalues are all 1). This fact leads to a linear equation in certain cosines of rational multiples of $\pi$. We find all solutions to this equation using a theorem of Conway and Jones [1976]. We then go on to find which of these solutions lie in parameter space, that is, which of the solutions lie outside the central triangle in Figure 1. As we have already indicated, it suffices to consider those $\tau$ whose argument lies in $\left[0, \frac{\pi}{3}\right]$. Such values of $\tau$ lying outside the central triangle and yet inside both the deltoid and circle are shown in Figure 2.

3.1. The eigenvalue equation. We now investigate when both $I_{1} I_{2}$ and $I_{1} I_{2} I_{3}$ are elliptic of finite order. In fact our proof will be valid when $I_{1} I_{2}$ is parabolic and yields a new proof of Theorem 1.1. We know that, $I_{1} J$ (and hence $I_{1} I_{2} I_{3}$ ) is elliptic of finite order if and only if

$$
\tau=\operatorname{tr}\left(I_{1} J\right)=e^{i \alpha}+e^{i \beta}+e^{-i \alpha-i \beta},
$$

where $\alpha$ and $\beta$ are rational multiples of $\pi$. Likewise for $I_{1} I_{2}$. In fact we know slightly more. Since the intersection of $L_{1}$ and $L_{2}$ is a (-1)-eigenvector for each of $I_{1}$ and $I_{2}$ it must be a $(+1)$-eigenvector for $I_{1} I_{2}$. Hence the eigenvalues of $I_{1} I_{2}$ are $1, e^{2 i \theta}$ and $e^{-2 i \theta}$. That is

$$
|\tau|^{2}-1=\operatorname{tr}\left(I_{1} I_{2}\right)=2 \cos (2 \theta)+1,
$$

where $\theta$ is a rational multiple of $\pi$. From Corollary 2.10 (iii) we see that, geometrically, $\theta$ is just the angle between $L_{1}$ and $L_{2}$. If $\theta=0$ then $I_{1} I_{2}$ is parabolic (or the identity) and we shall include this case in our analysis. 




Figure 2. An enlarged view of part of Figure 1 showing the values of $\tau$ in Theorem 3.7. Of the groups from Theorem 3.7 (ii) we have only plotted those that are discrete, as enumerated in Proposition 4.5 .

We solve (3-1) and (3-2) by eliminating $\tau$. That is, we seek $\theta, \alpha, \beta$ rational multiples of $\pi$ so that

$$
2 \cos (2 \theta)+2=|\tau|^{2}=3+2 \cos (\alpha-\beta)+2 \cos (\alpha+2 \beta)+2 \cos (-2 \alpha-\beta) .
$$

Rearranging, this becomes

$$
\frac{1}{2}=\cos (2 \theta)-\cos (\alpha-\beta)-\cos (\alpha+2 \beta)-\cos (-2 \alpha-\beta) .
$$

Notice that there is a certain amount of ambiguity in the solutions of this equation. Given one solution $\alpha-\beta, \alpha+2 \beta$ and $2 \alpha-\beta$ we obtain other solutions by a sequence of the following operations:

(i) permuting $\alpha-\beta, \alpha+2 \beta$ and $-2 \alpha-\beta$;

(ii) changing the sign of all three of them;

(iii) adding a multiple of $2 \pi$ to one of them and subtracting the same multiple of $2 \pi$ from another.

Using the fact that all three angles sum to zero, the net result of these ambiguities is to possibly complex conjugate $\tau$ and/or to multiply $\tau$ by a power of $\omega=e^{2 \pi i / 3}$. These are precisely the ambiguities in $\tau$ we already know about. For example, adding $2 \pi$ to $\alpha+2 \beta$ and subtracting $2 \pi$ from $-2 \alpha-\beta$ sends $\alpha$ to $\alpha+\frac{2 \pi}{3}$ and $\beta$ to $\beta+\frac{2 \pi}{3}$. Hence it sends $\tau$ to $\tau \omega$. Likewise, swapping $\alpha+2 \beta$ and $-2 \alpha-\beta$ sends $\alpha$ to $-\beta$ and $\beta$ to $-\alpha$. This has the effect of sending $\tau$ to $\bar{\tau}$.

The theorem that we use to find all solutions to (3-3) is the wonderful [Conway and Jones 1976, Theorem 7]: 
Theorem 3.1 [Conway and Jones 1976]. Suppose that we are given at most four distinct rational multiples of $\pi$ lying strictly between 0 and $\frac{\pi}{2}$ for which some rational linear combination of their cosines is rational, but no proper subsum has this property. Then this linear combination is proportional to one of the following:

(a) $\frac{1}{2}=\cos \frac{\pi}{3}$,

(b) $0=-\cos \phi+\cos \left(\phi-\frac{\pi}{3}\right)+\cos \left(\phi+\frac{\pi}{3}\right)$ where $0<\phi<\frac{\pi}{6}$,

(c) $\frac{1}{2}=\cos \frac{\pi}{5}-\cos \frac{2 \pi}{5}$,

(d) $\frac{1}{2}=\cos \frac{\pi}{7}-\cos \frac{2 \pi}{7}+\cos \frac{3 \pi}{7}$,

(e) $\frac{1}{2}=\cos \frac{\pi}{5}-\cos \frac{\pi}{15}+\cos \frac{4 \pi}{15}$,

(f) $\frac{1}{2}=-\cos \frac{2 \pi}{5}+\cos \frac{2 \pi}{15}-\cos \frac{7 \pi}{15}$,

(g) $\frac{1}{2}=\cos \frac{\pi}{7}+\cos \frac{3 \pi}{7}-\cos \frac{\pi}{21}+\cos \frac{8 \pi}{21}$,

(h) $\frac{1}{2}=\cos \frac{\pi}{7}-\cos \frac{2 \pi}{7}+\cos \frac{2 \pi}{21}-\cos \frac{5 \pi}{21}$,

(i) $\frac{1}{2}=-\cos \frac{2 \pi}{7}+\cos \frac{3 \pi}{7}+\cos \frac{4 \pi}{21}+\cos \frac{10 \pi}{21}$,

(j) $\frac{1}{2}=-\cos \frac{\pi}{15}+\cos \frac{2 \pi}{15}+\cos \frac{4 \pi}{15}-\cos \frac{7 \pi}{15}$.

We claim that we may find all solutions to (3-3) by inspection from this theorem. In order to see this, observe that by sending $\phi$ to $\pi-\phi$ we send $\cos \phi$ to $-\cos \phi$. Thus, by allowing the angles to lie in $(0, \pi)$, for each equation in Theorem 3.1 we arrange for all the signs in front of the cosines to be the same. We then look for three of the angles that (after possibly changing their signs) add up to a multiple of $2 \pi$. Once again, working modulo $2 \pi$, we adjust the angles so that they sum to zero. The resulting angles are $\alpha-\beta, \alpha+2 \beta$ and $-2 \alpha-\beta$. The trickiest cases are those where there are fewer than four angles listed in Theorem 3.1. One then has to use one of the following identities to reconstruct (3-3):

(k) $1=\cos 0$,

(l) $0=\cos \frac{\pi}{2}$,

(m) $0=\cos \phi+\cos (\pi-\phi)$ for some angle $\phi$.

Note the identity given in Theorem 3.1 (b) holds for all angles $\phi \in[0,2 \pi$ ). The condition $0<\phi<\frac{\pi}{6}$ was only there to ensure that the three angles are in $\left(0, \frac{\pi}{2}\right)$. Since we are adding multiples of $\pi$ to our angles and changing their signs where necessary, when using Theorem 3.1 (b) we allow $\phi$ to be any angle.

Hence, by inspection, we find the only candidates for $\alpha-\beta, \alpha+2 \beta,-2 \alpha-\beta$ solving (3-3) and we list them in the following table along with $\theta$ (we have ordered them so that $\alpha-\beta<\alpha+2 \beta<2 \alpha+\beta$ ). From this we find $\alpha, \beta$ and $\alpha+\beta$. In the 
last column we indicate which of the identities (a) to (m) we have used.

\begin{tabular}{|c|c|c|c|c|c|c|c|c|}
\hline & $2 \theta$ & $\alpha-\beta$ & $\alpha+2 \beta$ & $2 \alpha+\beta$ & $\alpha$ & $\beta$ & $\alpha+\beta$ & \\
\hline (i) & $\frac{2 \pi}{3}$ & $\pi-\frac{\phi}{2}$ & $\pi$ & $2 \pi-\frac{\phi}{2}$ & $\pi-\frac{\phi}{3}$ & $\frac{\phi}{6}$ & $\pi-\frac{\phi}{6}$ & (a), (k), (m) \\
\hline (ii) & $\phi$ & $\frac{\pi}{3}-\phi$ & $\frac{\pi}{3}+\phi$ & $\frac{2 \pi}{3}$ & $\frac{\pi}{3}-\frac{\phi}{3}$ & $\frac{2 \phi}{3}$ & $\frac{\pi}{3}+\frac{\phi}{3}$ & (a), (b) \\
\hline (iii) & $\frac{\pi}{3}$ & $\frac{\pi}{4}$ & $\frac{\pi}{2}$ & $\frac{3 \pi}{4}$ & $\frac{\pi}{3}$ & $\frac{\pi}{12}$ & $\frac{5 \pi}{12}$ & (a), (l), (m) \\
\hline (iv) & $\frac{\pi}{5}$ & $\frac{3 \pi}{10}$ & $\frac{2 \pi}{5}$ & $\frac{7 \pi}{10}$ & $\frac{\pi}{3}$ & $\frac{\pi}{30}$ & $\frac{11 \pi}{30}$ & (c), (m) \\
\hline (v) & $\frac{3 \pi}{5}$ & $\frac{\pi}{10}$ & $\frac{4 \pi}{5}$ & $\frac{9 \pi}{10}$ & $\frac{\pi}{3}$ & $\frac{7 \pi}{30}$ & $\frac{17 \pi}{30}$ & (c), (m) \\
\hline (vi) & $\frac{\pi}{2}$ & $\frac{2 \pi}{7}$ & $\frac{4 \pi}{7}$ & $\frac{6 \pi}{7}$ & $\frac{8 \pi}{21}$ & $\frac{2 \pi}{21}$ & $\frac{10 \pi}{21}$ & (d), (l) \\
\hline (vii) & $\frac{\pi}{2}$ & $\frac{\pi}{15}$ & $\frac{11 \pi}{15}$ & $\frac{4 \pi}{5}$ & $\frac{13 \pi}{45}$ & $\frac{2 \pi}{9}$ & $\frac{23 \pi}{45}$ & (e), (l) \\
\hline (viii) & $\frac{\pi}{2}$ & $\frac{7 \pi}{15}$ & $\frac{17 \pi}{15}$ & $\frac{8 \pi}{5}$ & $\frac{31 \pi}{45}$ & $\frac{2 \pi}{9}$ & $\frac{41 \pi}{45}$ & (f), (l) \\
\hline (ix) & $\frac{\pi}{7}$ & $\frac{\pi}{21}$ & $\frac{4 \pi}{7}$ & $\frac{13 \pi}{21}$ & $\frac{2 \pi}{9}$ & $\frac{11 \pi}{63}$ & $\frac{25 \pi}{63}$ & (g) \\
\hline (x) & $\frac{5 \pi}{7}$ & $\frac{5 \pi}{21}$ & $\frac{19 \pi}{21}$ & $\frac{8 \pi}{7}$ & $\frac{29 \pi}{63}$ & $\frac{2 \pi}{9}$ & $\frac{43 \pi}{63}$ & (h) \\
\hline (xi) & $\frac{3 \pi}{7}$ & $\frac{11 \pi}{21}$ & $\frac{25 \pi}{21}$ & $\frac{12 \pi}{7}$ & $\frac{47 \pi}{63}$ & $\frac{2 \pi}{9}$ & $\frac{61 \pi}{63}$ & (i) \\
\hline
\end{tabular}

We reiterate that each line in this table is a representative of several equivalent solutions. These are obtained by permutation, changing sign and adding a multiple of $\frac{2 \pi}{3}$ to both $\alpha$ and $\beta$. For example, the solution (iii) also corresponds to the following pair of solutions (reordered so that $\beta<\alpha$ ):

$$
\begin{aligned}
& \alpha=\frac{\pi}{3}+\frac{2 \pi}{3}=\pi, \quad \beta=\frac{\pi}{12}+\frac{2 \pi}{3}=\frac{3 \pi}{4}, \alpha+\beta=\frac{5 \pi}{12}+\frac{4 \pi}{3}=\frac{7 \pi}{4} ; \\
& \alpha=\frac{2 \pi}{3}-\frac{\pi}{12}=\frac{7 \pi}{12}, \quad \beta=\frac{2 \pi}{3}-\frac{\pi}{3}=\frac{\pi}{3}, \quad \alpha+\beta=\frac{4 \pi}{3}-\frac{5 \pi}{12}=\frac{11 \pi}{12} .
\end{aligned}
$$

We then write down

$$
\tau=\operatorname{tr}\left(I_{1} J\right)=e^{i \alpha}+e^{i \beta}+e^{-i \alpha-i \beta}, \quad \text { and } \quad \operatorname{tr}\left(I_{1} I_{2} I_{3}\right)=e^{3 i \alpha}+e^{3 i \beta}+e^{-3 i \alpha-3 i \beta}
$$

using this table. As indicated earlier, the parameters $\tau \omega^{j}$ and $\bar{\tau} \omega^{j}$ correspond to the same group as $\tau$. So in the case of (iii) where $\tau=e^{i \pi / 3}+e^{-i \pi / 6} 2 \cos \frac{\pi}{4}$ the two equivalent solutions listed above yield, respectively,

$$
\begin{aligned}
\tau & =-1+i 2 \cos \frac{\pi}{4}=e^{2 \pi i / 3}\left(e^{i \pi / 3}+e^{-i \pi / 6} 2 \cos \frac{\pi}{4}\right), \\
\tau & =e^{i \pi / 3}-e^{-i \pi / 6} 2 \cos \frac{\pi}{4}=e^{2 \pi i / 3}\left(e^{-i \pi / 3}+e^{i \pi / 6} 2 \cos \frac{\pi}{4}\right) .
\end{aligned}
$$

Evaluating $\tau$ from each line in the table gives the following result. We have kept the same labelling (i) to (xi) as in the table.

Proposition 3.2. Suppose that $I_{1} I_{2}$ and $I_{1} I_{2} I_{3}$ are both elliptic of finite order (or possibly $I_{1} I_{2}$ is parabolic). Up to complex conjugating $\tau$ and multiplying by a power of $\omega$, then one of the following is true:

(i) $\tau=-e^{-i \phi / 3}$ for some angle $\phi$ that is a rational multiple of $\pi$; 
(ii) $\tau=e^{2 i \phi / 3}+e^{-i \phi / 3}=e^{i \phi / 6} 2 \cos \frac{\phi}{2}$ for some angle $\phi$ that is a rational multiple of $\pi$;

(iii) $\tau=e^{i \pi / 3}+e^{-i \pi / 6} 2 \cos \frac{\pi}{4}$;

(iv) $\tau=e^{i \pi / 3}+e^{-i \pi / 6} 2 \cos \frac{\pi}{5}$;

(v) $\tau=e^{i \pi / 3}+e^{-i \pi / 6} 2 \cos \frac{2 \pi}{5}$;

(vi) $\tau=e^{2 \pi i / 7}+e^{4 \pi i / 7}+e^{8 \pi i / 7}$;

(vii) $\tau=e^{2 \pi i / 9}+e^{-i \pi / 9} 2 \cos \frac{2 \pi}{5}$;

(viii) $\tau=e^{2 \pi i / 9}+e^{-\pi i / 9} 2 \cos \frac{4 \pi}{5}$;

(ix) $\tau=e^{2 \pi i / 9}+e^{-i \pi / 9} 2 \cos \frac{2 \pi}{7}$;

(x) $\tau=e^{2 \pi i / 9}+e^{-i \pi / 9} 2 \cos \frac{4 \pi}{7}$;

(xi) $\tau=e^{2 \pi i / 9}+e^{-i \pi / 9} 2 \cos \frac{6 \pi}{7}$.

Observe that the first two cases of the above theorem include various elementary groups:

- Putting $\phi=0$ in (i) we obtain $\tau=-1$ which yields the elementary group of order 6 where $J=I_{1} I_{2}$. Multiplying by $\bar{\omega}$, we see that this value of $\tau$ is equivalent to $\tau=e^{i \pi / 3}$.

- Putting $\phi=0$ in (ii) we obtain $\tau=2$ which yields the elementary group of order 2 where $I_{1}=I_{2}=I_{3}$.

- Putting $\phi=\frac{\pi}{2}$ in (ii) gives $\tau=e^{\pi i / 3}(1-i)$.

These three groups will be important for our discussion of Theorem 3.7 (i) below. We shall discuss elementary groups in more detail in Section 4.1. Moreover, it is clear that the only solution with $\theta=0$ involves setting $\phi=0$ in part (ii). This gives a new proof of Schwartz's theorem, Theorem 1.1.

3.2. Solutions in parameter space. We now consider the values of $\tau$ found in Proposition 3.2 and we check which of them satisfy the conditions of Corollary 2.4. In other words, we find which of them lies in one of the regions in Figure 1 where the signature is $(2,1)$. Note that since $|\tau| \leq 2$ the only possibilities are that $H_{\tau}$ has signature $(2,1)$ or $(3,0)$. We state our results in terms of the signature of $H_{\tau}$. First, for $\tau$ given in part (i) $H_{\tau}$ has signature $(3,0)$ unless $\tau=-1$ (or $-\omega$ or $-\bar{\omega})$, when $H_{\tau}$ is degenerate. We now consider the other cases one by one.

Lemma 3.3. If $\tau=e^{2 i \phi / 3}+e^{-i \phi / 3}$ then $H_{\tau}$ has signature $(2,1)$ if and only if $0<\cos \phi<1$ and signature $(3,0)$ if and only if $-1 \leq \cos \phi<0$. When $\cos \phi=0$ or 1 then $H_{\tau}$ is degenerate. 
Proof. We have $|\tau|^{2}=2+2 \cos \phi$ and $\tau^{3}=e^{2 i \phi}+3 e^{i \phi}+3+e^{-i \phi}$. Therefore $6|\tau|^{2}-\tau^{3}-\bar{\tau}^{3}-8=6(2+2 \cos \phi)-2\left(2 \cos ^{2} \phi+4 \cos \phi+2\right)-8=4 \cos \phi-4 \cos ^{2} \phi$.

This is positive when $0<\cos \phi<1$ and negative when $-1 \leq \cos \phi<0$.

Lemma 3.4. If $\tau=e^{2 \pi i / 7}+e^{4 \pi i / 7}+e^{8 \pi i / 7}$ then $H_{\tau}$ has signature $(3,0)$.

Proof. We can rewrite $\tau$ as $\frac{1}{2}(-1+i \sqrt{7})$. Thus from Lemma 2.3, the eigenvalues of $H_{\tau}$ are

$$
2+\tau+\bar{\tau}=1, \quad 2+\tau \bar{\omega}+\bar{\omega} \tau=\frac{5+\sqrt{21}}{2}, \quad 2+\tau \omega+\bar{\tau} \bar{\omega}=\frac{5-\sqrt{21}}{2} .
$$

These are all positive.

Lemma 3.5. Suppose that $\tau=e^{\pi i / 3}+e^{-\pi i / 6} 2 \cos \phi$ for some $\phi$. Then $H_{\tau}$ is degenerate, that is it has determinant zero.

Proof. We have

$$
\tau \omega+\bar{\tau} \bar{\omega}=(-1+2 i \cos \phi)+(-1-2 i \cos \phi)=-2 .
$$

Therefore, using Lemma 2.3 we see that $H_{\tau}$ has an eigenvalue of 0 , and hence is degenerate.

Lemma 3.6. If $\tau=e^{2 \pi i / 9}+e^{-\pi i / 9} 2 \cos \phi$ for some $\phi$ then $H_{\tau}$ has signature $(2,1)$ if and only if $\cos 3 \phi<-\frac{1}{2}$ and signature $(3,0)$ if and only if $\cos 3 \phi>-\frac{1}{2}$. If $\cos 3 \phi=-\frac{1}{2}$ then $H_{\tau}$ is degenerate.

Proof. We have

$$
\begin{aligned}
|\tau|^{2} & =1+2 \cos \phi+4 \cos ^{2} \phi, \\
\tau^{3} & =e^{2 \pi i / 3}+e^{\pi i / 3} 6 \cos \phi+12 \cos ^{2} \phi+e^{-\pi i / 3} 8 \cos ^{3} \phi .
\end{aligned}
$$

Thus

$$
\begin{aligned}
6|\tau|^{2}-\tau^{3} & -\bar{\tau}^{3}-8 \\
& =6\left(1+2 \cos \phi+4 \cos ^{2} \phi\right)-\left(-1+6 \cos \phi+24 \cos ^{2} \phi+8 \cos ^{3} \phi\right)-8 \\
& =-1+6 \cos \phi-8 \cos ^{3} \phi=-1-2 \cos 3 \phi .
\end{aligned}
$$

This is positive if and only if $\cos 3 \phi<-\frac{1}{2}$ and negative if and only if $\cos 3 \phi>-\frac{1}{2}$.

We have shown that the values of $\tau$ given in parts (vi), (viii), (x) and (xi) of Proposition 3.2 correspond to values of $\tau$ for which $H_{\tau}$ has signature $(3,0)$. Thus they do not correspond to groups in $\mathrm{SU}(2,1)$. There are six values of $\tau$ where $H_{\tau}$ is degenerate, each of which has the form $\tau=e^{\pi i / 3}+e^{-\pi i / 6} 2 \cos \phi$. First, the three values with $\phi=\frac{\pi}{4}, \frac{\pi}{5}, \frac{2 \pi}{5}$ come from parts (iii), (iv) and (v) of Proposition 3.2. Secondly, there are the three values $\tau=e^{i \pi / 3}, 2, e^{i \pi / 3}(1-i)$ listed at the end of 
Section 3.1 and which correspond to elementary groups. These values correspond to $\phi=\frac{\pi}{2}, \frac{\pi}{6}, \frac{\pi}{3}$.

We now summarize the results of this section.

Theorem 3.7. Let $I_{1}, I_{2}=J I_{1} J^{-1}$ and $I_{3}=J^{-1} I_{1} J$ be involutions in $\operatorname{SU}(2,1)$ each fixing a complex line, where $J \in \mathrm{SU}(2,1)$ has order three. Suppose that $I_{1} I_{2}$ and $I_{1} I_{2} I_{3}=\left(I_{1} J\right)^{3}$ both have finite order. If $\Delta=\left\langle I_{1}, I_{2}, I_{3}\right\rangle$ is discrete then, up to complex conjugating or multiplying by $\omega$ or $\bar{\omega}$, one of the following is true:

(i) $\tau=e^{\pi i / 3}+e^{-\pi i / 6} 2 \cos \frac{2 \pi}{n}=e^{i \pi / 3}\left(1-2 i \cos \frac{2 \pi}{n}\right)$ where $n=4,5,6,8,10$ or 12 ;

(ii) $\tau=e^{2 i \phi / 3}+e^{-i \phi / 3}$ where $\phi$ is a rational multiple of $\pi$ in $\left(0, \frac{\pi}{2}\right)$;

(iii) $\tau=e^{2 \pi i / 9}+e^{-\pi i / 9} 2 \cos \frac{2 \pi}{5}$;

(iv) $\tau=e^{2 \pi i / 9}+e^{-\pi i / 9} 2 \cos \frac{2 \pi}{7}$.

Note that the cases $n=6$ and $n=12$ in (i) correspond to the endpoints of the open interval given in (ii).

\section{The discrete groups}

In this section we analyze all the groups from Theorem 3.7. We show that those groups listed in parts (i), (iii) and (iv) are all discrete and that finitely many of those given in (ii) are discrete. The parameter values corresponding to discrete groups are all plotted in Figure 2.

4.1. The elementary groups. In this section we consider what happens when $\tau=$ $e^{\pi i / 3}+e^{-\pi i / 6} 2 \cos \phi=e^{i \pi / 3}(1-2 i \cos \phi)$ where $\phi=\frac{2 \pi}{n}$ for $n=4,5,6,8,10$ or 12. These are the groups from Proposition 3.2 (iii), (iv), (v) together with the cases where $\tau=e^{i \pi / 3}, \tau=2$ and $\tau=e^{\pi i / 3}(1-i)$. These are the six parameter values listed in Theorem 3.7 (i).

When $\tau=2$ all three complex lines coincide and so $J$ maps $L_{1}$ to itself, fixing a single point of $L_{1}$. Moreover, $I_{1}$ and $J$ commute and $\left\langle I_{1}, J\right\rangle$ is a cyclic group of order six, generated by $I_{1} J$.

In each case $\tau$ satisfies $\tau \omega+\bar{\tau} \bar{\omega}=-2$ which is one of the three linear factors of the equation $6|\tau|^{2}-\tau^{3}-\bar{\tau}^{3}-8=0$. Thus, by Lemma 2.2 the polar vectors $\mathbf{n}_{1}, \mathbf{n}_{2}$ and $\mathbf{n}_{3}$ are linearly dependent. In all cases, except for $\tau=2$, we can write down matrix representatives for $I_{1}, I_{2}, I_{3}$ and $J$ as was done at the very end of Section 2.2. These matrices are block diagonal, the upper left hand block lying in a copy of $\mathrm{U}(2)$ (preserving the Hermitian form given by the upper left hand $2 \times 2$ block of $H_{\tau}^{\prime}$ ). We can multiply them by scalars so that the upper left hand block 
has determinant 1 . This yields

$i I_{1}=\left(\begin{array}{ccc}i & i \tau & 0 \\ 0 & -i & 0 \\ 0 & 0 & -i\end{array}\right), \omega J=\left(\begin{array}{ccc}0 & -\bar{\omega} & 0 \\ \omega & -1 & 0 \\ 0 & 0 & 1\end{array}\right), i \omega I_{1} J=\left(\begin{array}{ccc}-2 \cos \phi-i & 2 \bar{\omega} \cos \phi & 0 \\ -i \omega & i & 0 \\ 0 & 0 & -i\end{array}\right)$

where we have used $\tau=e^{\pi i / 3}(1-2 i \cos \phi)=-\bar{\omega}+2 i \bar{\omega} \cos \phi$. By examining the traces of the upper left hand blocks of $i I_{1}, \omega J$ and $i \omega I_{1} J$ we see that they form a dihedral $(n=4)$, tetrahedral $(n=6)$, octahedral $(n=8)$ or icosahedral $(n=5,10)$ group. Therefore $\left\langle I_{1}, J\right\rangle$ is a finite central extension of such a group and hence, in each case, is finite.

We remark that in each case $\operatorname{tr}\left(I_{j} I_{j+1}\right)=4 \cos ^{2} \phi=1+2 \cos (2 \theta)$. We could read off the values of $\theta$ from the table given earlier or we can calculate them directly:

\begin{tabular}{llll}
$\phi$ & $4 \cos ^{2} \phi$ & $2 \cos (2 \theta)$ & $2 \theta$ \\
\hline$\frac{\pi}{2}$ & 0 & -1 & $\frac{2 \pi}{3}$ \\
$\frac{2 \pi}{5}$ & $\frac{1}{2}(3-\sqrt{5})$ & $\frac{1}{2}(1-\sqrt{5})$ & $\frac{3 \pi}{5}$ \\
$\frac{\pi}{3}$ & 1 & 0 & $\frac{\pi}{2}$ \\
$\frac{\pi}{4}$ & 2 & 1 & $\frac{\pi}{3}$ \\
$\frac{\pi}{5}$ & $\frac{1}{2}(3+\sqrt{5})$ & $\frac{1}{2}(1+\sqrt{5})$ & $\frac{\pi}{5}$ \\
$\frac{\pi}{6}$ & 3 & 2 & 0
\end{tabular}

4.2. The groups with $\tau=e^{2 \pi i / 9}+e^{-\pi i / 9} 2 \cos \phi$. In this section we consider the equilateral triangle groups corresponding to $\tau=e^{2 \pi i / 9}(1-2 \omega \cos \phi)$ where $\omega=e^{2 \pi i / 3}$ is a cube root of unity and $\phi=\frac{2 \pi}{5}$ or $\frac{2 \pi}{7}$. We remark that when $\phi=\frac{2 \pi}{6}$ we obtain $\tau=e^{2 \pi i / 9}(1-2 \omega \cos \phi)=e^{2 \pi i / 9}+e^{-\pi i / 3}$ which is one of the groups from Theorem 3.7 (ii) and is treated in Section 4.3. We want to eliminate the factor of $e^{2 \pi i / 9}$ from our matrix entries. Therefore we apply the matrix $C=\operatorname{diag}\left(e^{-2 \pi i / 9}, 1, e^{2 \pi i / 9}\right)$ to the $\mathbf{n}_{j}$, and hence to the whole set-up. The images of the polar vectors under $C$ are

$$
\mathbf{n}_{1}=\left(\begin{array}{c}
e^{-2 \pi i / 9} \\
0 \\
0
\end{array}\right), \quad \mathbf{n}_{2}=\left(\begin{array}{l}
0 \\
1 \\
0
\end{array}\right), \quad \mathbf{n}_{3}=\left(\begin{array}{c}
0 \\
0 \\
e^{2 \pi i / 9}
\end{array}\right) .
$$

The matrix $N$ whose columns are $\mathbf{n}_{1}, \mathbf{n}_{2}$ and $\mathbf{n}_{3}$ is simply $C$. Therefore, the new Hermitian form, which we call $H_{\phi}$, satisfies $C^{*} H_{\phi} C=H_{\tau}$. Clearly $C^{*}=C^{-1}$ and so

$$
H_{\phi}=C H_{\tau} C^{-1}=\left(\begin{array}{ccc}
2 & 1-2 \omega \cos \phi & \bar{\omega}-2 \omega \cos \phi \\
1-2 \bar{\omega} \cos \phi & 2 & 1-2 \omega \cos \phi \\
\omega-2 \bar{\omega} \cos \phi & 1-2 \bar{\omega} \cos \phi & 2
\end{array}\right)
$$


Obviously, since $H_{\tau}$ and $H_{\phi}$ are conjugate, they have the same eigenvalues. Using Lemma 3.6 we immediately have

Lemma 4.1. When $\phi=\frac{2 \pi}{5}$ or $\phi=\frac{2 \pi}{7}$ the matrix $H_{\phi}$ has signature $(2,1)$. When $\phi=\frac{4 \pi}{5}, \frac{4 \pi}{7}$ or $\frac{6 \pi}{7}$ the matrix $H_{\phi}$ has signature $(3,0)$.

After conjugating by $C$, the complex involutions are given by

$$
\begin{aligned}
I_{1} & =\left(\begin{array}{ccc}
1 & 1-2 \omega \cos \phi & \bar{\omega}-2 \omega \cos \phi \\
0 & -1 & 0 \\
0 & 0 & -1
\end{array}\right), \\
I_{2} & =\left(\begin{array}{ccc}
-1 & 0 & 0 \\
1-2 \bar{\omega} \cos \phi & 1 & 1-2 \omega \cos \phi \\
0 & 0 & -1
\end{array}\right), \\
I_{3} & =\left(\begin{array}{ccc}
-1 & 0 & 0 \\
0 & -1 & 0 \\
\omega-2 \bar{\omega} \cos \phi & 1-2 \bar{\omega} \cos \phi & 1
\end{array}\right) .
\end{aligned}
$$

The entries of $I_{1}, I_{2}$ and $I_{3}$ all have determinant one and lie in the ring $\mathbb{Z}[\omega, 2 \cos \phi]$. It is standard to write $\mathrm{SU}(H ; \mathbb{O})$ for the group of unimodular matrices preserving the Hermitian form $H$ whose entries lie in the ring $\mathcal{O}$. Thus

Lemma 4.2. The group $\Delta=\left\langle I_{1}, I_{2}, I_{3}\right\rangle$ generated by the matrices given in (4-1), (4-2) and (4-3) is a subgroup of $\mathrm{SU}\left(H_{\phi} ; \mathbb{Z}[\omega, 2 \cos \phi]\right)$.

Since $\omega$ and $2 \cos \frac{2 \pi}{n}$ are both algebraic integers, we see that every element of the ring $\mathbb{Z}\left[\omega, 2 \cos \frac{2 \pi}{n}\right]$ is an algebraic integer in $\mathbb{Q}\left(\omega, 2 \cos \frac{2 \pi}{n}\right)$. The field $\mathbb{Q}\left(\omega, 2 \cos \frac{2 \pi}{n}\right)$ is a totally imaginary quadratic extension of the totally real number field $\mathbb{Q}\left(2 \cos \frac{2 \pi}{n}\right)$. The following result (in the case $\left.\phi=\frac{2 \pi}{5}\right)$ is essentially identical to that given in [Deraux 2006, Corollary 2.6] and is similar to the proof of [Schwartz 2003, Corollary 1.4].

Proposition 4.3. For $\phi=\frac{2 \pi}{5}$ or $\phi=\frac{2 \pi}{7}$ the group $\mathrm{SU}\left(H_{\phi} ; \mathbb{Z}[\omega, 2 \cos \phi]\right)$ is arithmetic and hence discrete. In particular, $\Delta$ is discrete.

Proof. We give the proof in the case of $n=7$. The proof for $n=5$ is almost the same; see also [Deraux 2006, Corollary 2.6].

The field $\mathbb{Q}\left(\omega, 2 \cos \frac{2 \pi}{7}\right)$ is a totally imaginary quadratic extension of the totally real number field $\mathbb{Q}\left(2 \cos \frac{2 \pi}{7}\right)$. Let $\mathbb{Q}\left(c^{\prime}\right)$ be the totally real number field obtained from any nontrivial Galois conjugate of $c^{\prime}$ of $2 \cos \frac{2 \pi}{7}$ and let $\mathbb{Q}\left(\omega, c^{\prime}\right)$ be a compatible quadratic extension corresponding to $\mathbb{Q}(\omega, c)$. The only Galois conjugates of $2 \cos \frac{2 \pi}{7}$ are $2 \cos \frac{4 \pi}{7}$ and $2 \cos \frac{6 \pi}{7}$. 
Let $H_{4 \pi / 7}$ and $H_{6 \pi / 7}$ be the Hermitian forms obtained by applying these Galois automorphisms to $H_{2 \pi / 7}$. From Lemma 4.1 we see that $H_{4 \pi / 7}$ and $H_{6 \pi / 7}$ are positive definite. Therefore the corresponding groups $\mathrm{SU}\left(H_{4 \pi / 7}\right)$ and $\mathrm{SU}\left(H_{6 \pi / 7}\right)$ are compact.

Let $x \in \mathbb{Z}\left[\omega, 2 \cos \frac{2 \pi}{7}\right]$. Then $x$ is an algebraic integer in $\mathbb{Q}\left(\omega, 2 \cos \frac{2 \pi}{7}\right)$. Let $x^{\prime}$ and $x^{\prime \prime}$ be its Galois conjugates in $\mathbb{Q}\left(\omega, 2 \cos \frac{4 \pi}{7}\right)$ and $\mathbb{Q}\left(\omega, 2 \cos \frac{6 \pi}{7}\right)$. The map $x \mapsto\left(x, x^{\prime}, x^{\prime \prime}\right)$ maps $\mathbb{Z}\left[\omega, 2 \cos \frac{2 \pi}{7}\right]$ to a discrete subset of $\mathbb{C}^{3}$. Hence

$$
\begin{aligned}
\mathrm{SU}\left(H_{2 \pi / 7} ; \mathbb{Z}\left[\omega, 2 \cos \frac{2 \pi}{7}\right]\right) & \underset{\times \mathrm{SU}\left(H_{4 \pi / 7} ; \mathbb{Z}\left[\omega, 2 \cos \frac{4 \pi}{7}\right]\right)}{\times \operatorname{SU}\left(H_{6 \pi / 7} ; \mathbb{Z}\left[\omega, 2 \cos \frac{6 \pi}{7}\right]\right)}
\end{aligned}
$$

is discrete. Since $\mathrm{SU}\left(H_{4 \pi / 7}\right)$ and $\mathrm{SU}\left(H_{6 \pi / 7}\right)$ are compact, the image of projection onto the first factor, namely $\mathrm{SU}\left(H_{2 \pi / 7} ; \mathbb{Z}\left[\omega, 2 \cos \frac{2 \pi}{7}\right]\right)$ is also discrete.

Note that the groups we eliminated from Proposition 3.2 (viii), (x) and (xi) using Lemma 3.6 are just the Galois conjugates of the two groups we are considering.

Proposition 4.4. The group with $\tau=e^{2 \pi i / 9}+e^{-\pi i / 9} 2 \cos \frac{2 \pi}{5}$ is Deraux's lattice.

Proof. We calculate that $|\tau|^{2}=1+2 \cos \frac{2 \pi}{5}+4 \cos ^{2} \frac{2 \pi}{5}=2$. Hence $I_{1} I_{2}$ has order 4. The eigenvalues of $I_{1} I_{2} I_{3}$ are $\left(e^{2 \pi i / 9}\right)^{3}=\omega,\left(e^{-\pi i / 9 \pm 2 \pi i / 5}\right)^{3}=\omega e^{ \pm i \pi / 5}$. These are the same as the eigenvalues of $I_{1} I_{2} I_{3}$ found in [Deraux 2006, Eq. (2.15)]. Thus the groups are the same.

We now briefly discuss the group with $\tau=e^{2 \pi i / 9}+e^{-\pi i / 9} 2 \cos \frac{2 \pi}{7}$. This does not seem to have previously appeared in the literature. It is easy to show that $\operatorname{tr}\left(I_{1} I_{2}\right)=1+2 \cos \frac{\pi}{7}$ and so $I_{1} I_{2}$ has order 14. Furthermore, $\operatorname{tr}\left(I_{1} I_{2} I_{1} I_{3}\right)=$ $2 \cos \frac{\pi}{7}+2>3$ and so $I_{1} I_{2} I_{1} I_{3}$ is loxodromic. Since $I_{1} I_{2} I_{3}$ is elliptic, this means that, in the language of [Schwartz 2002], $\Delta$ is of Type B; see also [Parker 2006, Proposition 7.5] for other discrete, unfaithful groups of Type B. It is not clear whether or not $\Delta$ is the whole of the lattice $\operatorname{SU}\left(H_{7} ; \mathbb{Z}\left[\omega, 2 \cos \frac{2 \pi}{7}\right]\right)$. The fact that $I_{1} I_{2} I_{1} I_{3}$ is loxodromic would indicate that, in fact, $\Delta$ may not be a lattice. This group merits further investigation.

4.3. Subgroups of Livnés lattices. We consider the groups for which

$$
\tau=e^{2 i \phi / 3}+e^{-i \phi / 3}=e^{i \phi / 6} 2 \cos \frac{\phi}{2}
$$

for some angle $\phi \in\left(0, \frac{\pi}{2}\right)$. Note that we have already treated the cases of $\phi=0$ and $\phi=\frac{\pi}{2}$ in Section 4.1. The main result of this section is the following: 
Proposition 4.5. Let $\tau=e^{2 i \phi / 3}+e^{-i \phi / 3}$ for some angle $\phi \in\left(0, \frac{\pi}{2}\right)$. Then the group $\left\langle I_{1}, I_{1}, I_{3}\right\rangle$ is discrete if and only if $\phi=2 \pi / p$ where $p=5,6,7,8,9,10,12$ or 18.

We remark that if $p=6$ then $I_{1} I_{2} I_{3}$ is parabolic and so we are not in the case considered in Theorem 3.7. In this case it is particularly easy to prove discreteness and we include it for completeness. As we remarked in Section 4.2, when $p=6$ we can write $\tau=e^{2 \pi i / 9}(1-2 \omega \cos \phi)=e^{2 \pi i / 9}(1-\omega)$. We can then conjugate $I_{1}, I_{2}$ and $I_{3}$ into the forms (4-1), (4-2) and (4-3) respectively. These matrices all have entries in the ring of Eisenstein integers $\mathbb{Z}[\omega]$, as does every matrix in $\Delta=\left\langle I_{1}, I_{2}, I_{3}\right\rangle$. Since $\mathbb{Z}[\omega]$ is a discrete subring of $\mathbb{C}$ we see that $\Delta$ is discrete; see [Falbel and Parker 2006] for a more detailed discussion of this group.

We begin by showing that if $\phi$ does not take one of the values listed in Proposition 4.5 then $\Delta=\left\langle I_{1}, I_{2}, I_{3}\right\rangle$ cannot be discrete. We do this by showing that the subgroup $\left\langle I_{1}, I_{1} I_{2} I_{3}\right\rangle$ of $\Delta$ is not discrete for these values of $\tau$. In this discussion we exclude the case of $\phi=\frac{\pi}{3}$ (that is $p=6$ ) which we have already discussed.

We can see from line (ii) of the table given in Section 3.1 that the eigenvalues of $I_{1} J$ are

$$
e^{i \alpha}=e^{i(\pi-\phi) / 3}, \quad e^{i \beta}=e^{2 i \phi / 3} \quad \text { and } \quad e^{-i(\alpha+\beta)}=e^{i(-\pi-\phi) / 3} .
$$

Hence the eigenvalues of $I_{1} I_{2} I_{3}=\left(I_{1} J\right)^{3}$ are

$$
e^{3 i \alpha}=-e^{-i \phi}, \quad e^{3 i \beta}=e^{2 i \phi} \quad \text { and } \quad e^{-3 i(\alpha+\beta)}=-e^{-i \phi} .
$$

Therefore in this case $I_{1} I_{2} I_{3}$ has a repeated eigenvalue and so is a complex reflection with rotation angle $\pi-3 \phi$; it cannot be parabolic as it is the cube of an elliptic map. (Note that when $\phi=\frac{2 \pi}{6}$ then $I_{1} J$ has a repeated eigenvalue and is parabolic, as is $I_{1} I_{2} I_{3}$.) By examining the eigenvectors, one can show that when $\phi<\frac{2 \pi}{6}$ then $I_{1} I_{2} I_{3}$ is complex reflection in a complex line and when $\phi>\frac{2 \pi}{6}$ then $I_{1} I_{2} I_{3}$ is complex reflection in a point. We will give the details in the former case. The latter case is almost identical.

Lemma 4.6. Let $\tau=e^{2 i \phi / 3}+e^{-i \phi / 3}$ with $0<\phi<\frac{\pi}{3}$. Let $L_{1}$ and $L_{123}$ be the complex lines fixed by $I_{1}$ and $I_{1} I_{2} I_{3}$ respectively. These complex lines are ultraparallel and their common orthogonal $L^{\perp}$ is preserved by the group $\left\langle I_{1}, I_{1} I_{2} I_{3}\right\rangle$. This group acts on $L^{\perp}$ as the index 2 holomorphic subgroup of the group generated by reflections in the sides of a hyperbolic triangle with angles $\frac{\pi}{2}, \frac{\phi}{2}, \frac{1}{2}(\pi-3 \phi)$.

Proof. Since $I_{1}$ and $I_{1} I_{2} I_{3}$ are complex reflections, they preserve all complex lines orthogonal to $L_{1}$ and $L_{123}$, respectively. Suppose that $L_{1}$ and $L_{123}$ are not ultraparallel. Let their intersection be $\mathbf{z}$. Then $\mathbf{z}$ is fixed by $I_{1}$ and by $I_{1} I_{2} I_{3}$. Hence it is also fixed by $I_{2} I_{3}$ and so must be $L_{2} \cap L_{3}$. In other words, $\mathbf{z}$ is fixed by $I_{1}, I_{2}$ 
and $I_{3}$ and hence the group must be elementary. This is a contradiction to Lemmas 2.2 and 3.3.

Hence $L_{1}$ and $L_{123}$ are ultraparallel. Their common orthogonal is preserved by $I_{1}$ and $I_{1} I_{2} I_{3}$ which act as rotations through angles $\pi$ and $\pi-3 \phi$ respectively. Moreover $L^{\perp}$ is preserved by $I_{2} I_{3}$ and so its polar vector must be an eigenvector of $I_{2} I_{3}$. The eigenvalues of $I_{2} I_{3}$ corresponding to positive vectors are $e^{i \phi}$ and $e^{-i \phi}$. Hence $I_{2} I_{3}$ acts on $L^{\perp}$ as a rotation through $\phi$.

In the case where $\frac{\pi}{3}<\phi<\frac{\pi}{2}$ then $I_{1} I_{2} I_{3}$ is complex reflection in a point. The complex line $L^{\perp}$ is now the complex line through this point orthogonal to $L_{1}$. A similar argument shows that $\left\langle I_{1}, I_{1} I_{2} I_{3}\right\rangle$ acts on $L^{\perp}$ as the index 2 holomorphic subgroup of the group generated by reflections in the sides of a hyperbolic triangle with angles $\frac{\pi}{2}, \frac{\phi}{2}, \frac{1}{2}(3 \phi-\pi)$. We can now use plane hyperbolic geometry to complete the proof of Proposition 4.5.

Proposition 4.7. Let $\tau=e^{2 i \phi / 3}+e^{-i \phi / 3}$ with $0<\phi<\frac{\pi}{2}$ and $\phi \neq \frac{\pi}{3}$. The group $\left\langle I_{1}, I_{1} I_{2} I_{3}\right\rangle$ is discrete if and only if $\phi=\frac{2 \pi}{p}$ for $p=5,7,8,9,10,12$ or 18.

Proof. We consider the subgroup $\left\langle I_{1}, I_{1} I_{2} I_{3}\right\rangle$ and its action on $L^{\perp}$. This group is generated by two elliptic maps whose product is also elliptic. [Knapp 1968, Theorem 2.3] has characterized when such a group is discrete. Our case is particularly easy because $I_{1}$ has order 2 and so one of the angles in our triangle is a right angle. Hence if $\left\langle I_{1}, I_{1} I_{2} I_{3}\right\rangle$ is discrete then we are in Case I or Case IV of Knapp's theorem. In other words either $\frac{\phi}{2}=\frac{\pi}{p}$ and $\frac{1}{2}|\pi-3 \phi|=\frac{\pi}{d}$ or else one of $\frac{\phi}{2}$ and $\frac{1}{2}|\pi-3 \phi|$ equals $\frac{\pi}{m}$ and the other equals $\frac{2 \pi}{m}$ for some odd integer $m$. In fact, if we solve for $m$ in the last case we see that either $m=10$ or $m=14$, neither of which is odd. Therefore we must have $\phi=\frac{2 \pi}{p}$ and $|\pi-3 \phi|=\frac{\pi}{p}|p-6|=\frac{2 \pi}{d}$. The values of $p$ in the proposition are precisely those $\left(\frac{2 \pi}{p}<\frac{\pi}{2}\right)$ for which $\frac{2 p}{|p-6|}$ is an integer $d$.

This shows that when $\phi$ is not one of the given values then the group $\Delta$ is not discrete. It remains to show that the values of $\phi$ listed in Proposition 4.5 do indeed correspond to discrete groups. This follows immediately from the following result and will complete the proof of Proposition 4.5.

Proposition 4.8 [Parker 2006, Corollary 7.4]. Let $\tau=e^{2 i \phi / 3}+e^{-i \phi / 3}$. When $\phi=\frac{2 \pi}{p}$ for $p=5,6,7,8,9,10,12$ or 18 the group $\Delta=\left\langle I_{1}, I_{2}, I_{3}\right\rangle$ is discrete. Moreover, when $p=5$ this group is a cocompact lattice in $\mathrm{SU}(2,1)$, when $p=6,7,8,9,10$, 12 or 18 it is geometrically infinite.

We now give a brief discussion of how one might prove Proposition 4.8. In [Parker 2006], this result is proved by demonstrating that, for such $\phi$, the group $\Delta$ is a normal subgroup of one of the lattices first described by Livné [1981]. There are several ways to show that Livné's groups are discrete. For example, fundamental 
domains for these groups were constructed in [Parker 2006], and presentations were given using Poincaré's polyhedron theorem. Alternatively, one could relate such a group to one of Mostow's ball 5-tuples and then use his discreteness criterion $\Sigma$ INT [Mostow 1986]; see also [Deligne and Mostow 1993, Theorem 16.1]. The fact that this criterion precisely characterizes discreteness is due to Sauter [1990], who analyzed the few remaining cases not treated by Mostow.

Alternatively, for $p=5,6,7,8,10,12,18$ we could show that $\Delta$ is arithmetic and hence discrete using a similar argument to Proposition 4.3. In doing this we use Lemma 3.3 to show that $H_{\tau}$ has signature $(2,1)$ if and only if $0<\cos \phi<1$. This argument does not work when $p=9$. The Galois conjugates of $2 \cos \frac{2 \pi}{9}$ are $2 \cos \frac{4 \pi}{9}$ and $2 \cos \frac{6 \pi}{9}$. Because $\cos \frac{4 \pi}{9}>0$, Lemma 3.3 implies that the corresponding Hermitian form has signature $(2,1)$. In fact when $p=9$ the group $\Delta$ is nonarithmetic and, in this case, one must use a geometrical argument.

Furthermore, there are other ways of showing that values of $\tau$ listed in Proposition 4.5 are the only ones of this form that correspond to discrete groups. We could again use Mostow's $\Sigma$ INT condition. A more direct approach would be to use the complex hyperbolic Jørgensen's inequality [Jiang et al. 2003] to show that when $\phi$ is not one of the angles listed above then $\Delta$ is not discrete.

\section{Acknowledgements}

I would like to thank Martin Deraux, Elisha Falbel and Ben McReynolds for useful conversations and Pierre Will for drawing the figures. I am very grateful to the referee for reading the paper carefully and for giving me many useful suggestions.

\section{References}

[Conway and Jones 1976] J. H. Conway and A. J. Jones, "Trigonometric Diophantine equations (On vanishing sums of roots of unity)", Acta Arith. 30:3 (1976), 229-240. MR 54 \#10141 Zbl 0349. 10014

[Deligne and Mostow 1993] P. Deligne and G. D. Mostow, Commensurabilities among lattices in PU $(1, n)$, Annals of Mathematics Studies 132, Princeton University Press, Princeton, NJ, 1993. MR 95a:22013 Zbl 0826.22011

[Deraux 2006] M. Deraux, "Deforming the $\mathbb{R}$-Fuchsian (4, 4, 4)-triangle group into a lattice", Topology 45:6 (2006), 989-1020. MR 2007m:32015 Zbl 1120.20052

[Falbel and Parker 2006] E. Falbel and J. R. Parker, "The geometry of the Eisenstein-Picard modular group”, Duke Math. J. 131:2 (2006), 249-289. MR 2007f:22011 Zbl 1109.22007

[Goldman 1999] W. M. Goldman, Complex hyperbolic geometry, Clarendon, New York, 1999. MR 2000g:32029 Zbl 0939.32024

[Goldman and Parker 1992] W. M. Goldman and J. R. Parker, "Complex hyperbolic ideal triangle groups”, J. Reine Angew. Math. 425 (1992), 71-86. MR 93c:20076 Zbl 0739.53055

[Jiang et al. 2003] Y. Jiang, S. Kamiya, and J. R. Parker, “Jørgensen’s inequality for complex hyperbolic space”, Geom. Dedicata 97 (2003), 55-80. MR 2004i:22012 Zbl 1037.20053 
[Knapp 1968] A. W. Knapp, "Doubly generated Fuchsian groups", Michigan Math. J. 15 (1968), 289-304. MR 40 \#1483 Zbl 0167.07002

[Livné 1981] R. A. Livné, On certain covers of the universal elliptic curve, $\mathrm{PhD}$ thesis, Harvard University, 1981.

[Mostow 1980] G. D. Mostow, "On a remarkable class of polyhedra in complex hyperbolic space", Pacific J. Math. 86:1 (1980), 171-276. MR 82a:22011 Zbl 0456.22012

[Mostow 1986] G. D. Mostow, "Generalized Picard lattices arising from half-integral conditions", Inst. Hautes Études Sci. Publ. Math. 63 (1986), 91-106. MR 88a:22023b Zbl 0615.22009

[Parker 2006] J. R. Parker, "Cone metrics on the sphere and Livné's lattices", Acta Math. 196:1 (2006), 1-64. MR 2007j:32021 Zbl 1100.57017

[Parker and Paupert 2007] J. R. Parker and J. Paupert, "Unfaithful complex hyperbolic triangle groups II: Higher order reflections", available at http://www.math.utah.edu/ paupert/GenTri2.pdf, preprint, 2007

[Pratoussevitch 2005] A. Pratoussevitch, "Traces in complex hyperbolic triangle groups", Geom. Dedicata 111 (2005), 159-185. MR 2006d:32036 Zbl 1115.32015

[Sauter 1990] J. K. Sauter, Jr., "Isomorphisms among monodromy groups and applications to lattices in PU(1, 2)”, Pacific J. Math. 146:2 (1990), 331-384. MR 92d:22016 Zbl 0759.22013

[Schwartz 2001] R. E. Schwartz, "Ideal triangle groups, dented tori, and numerical analysis", Ann. of Math. (2) 153:3 (2001), 533-598. MR 2002j:57031 Zbl 1055.20040

[Schwartz 2002] R. E. Schwartz, "Complex hyperbolic triangle groups", pp. 339-349 in Proceedings of the International Congress of Mathematicians (Beijing, 2002), vol. II, edited by T. Li, Higher Education Press, Beijing, 2002. MR 2004b:57002 Zbl 1022.53034

[Schwartz 2003] R. E. Schwartz, "Real hyperbolic on the outside, complex hyperbolic on the inside”, Invent. Math. 151:2 (2003), 221-295. MR 2003j:32032 Zbl 1039.32030

[Schwartz 2007] R. E. Schwartz, Spherical CR geometry and Dehn surgery, Annals of Mathematics Studies 165, Princeton University Press, Princeton, NJ, 2007. MR 2286868 Zbl 1116.57016

Received November 28, 2007. Revised June 18, 2008.

JOHN R. PARKER

DePartMENT OF MATHEMATICAL SCIENCES

DURHAM UNIVERSITY

SOUTH ROAD

DURHAM DH1 3LE

UNITED KINGDOM

j.r.parker@durham.ac.uk

http://www.maths.dur.ac.uk/ dma0jrp/ 